\title{
Cryptanalysis of Two Efficient HIBE Schemes in the Standard Model
}

\author{
Xu An Wang and Xiaoyuan Yang \\ Key Laboratory of Information and Network Security \\ Engineering College of Chinese Armed Police Force, P.R. China \\ wangxahq@yahoo.com.cn
}

\begin{abstract}
In Informatica 32 (2008), Ren and Gu proposed an anonymous hierarchical identity based encryption scheme based on the q-ABDHE problem with full security in the standard model. Later in Indocrypt'08, they proposed another secure hierarchical identity based encryption scheme based on the q-TBDHE problem with full security in the standard model. They claimed that their schemes have short parameters, high efficiency and tight reduction. However, in this paper we give attacks to show their schemes are insecure at all. Concretely, from any first level private key, the adversary can easily derive a proper "private key" which can decrypt any ciphertexts for the target identity. That is to say, one key generation query on any first level identity excluding the target's first level identity, is enough to break their schemes ${ }^{1}$.
\end{abstract}

\section{Introduction}

\subsection{Backgroud}

IBE. In 1984, Shamir [13] first proposed the concept of identity based encryption (IBE) to simplify the certificate management. In traditional public key encryption (PKE) cryptosystem, a user's public key need to be certified by an authority CA to ensure its validity. Therefore, management of certificates is heavy for PKE. However, in an IBE cryptosystem, a user's public key can be represented as an arbitrary string, certificate management can be greatly simplified. Due to this benefit, IBE attracts great attention from the cryptography community. However, the first practical IBE scheme only realized by Boneh and Franklin in 2001 by using bilinear pairings [5]. In Eurocrypt'04, Boneh and Boyen proposed two new efficient selective identity secure (the attacker must commit the target identity before attack) IBE schemes without random oracles $\left(\mathrm{BB}_{1} \mathrm{IBE}\right.$ and $\left.\mathrm{BB}_{2} \mathrm{IBE}\right)$ [2]. Later Boneh and Boyen [3], Waters [14] proposed new IBE schemes with full security (the attacker can adaptively choose the target identity). In Eurocrypt'06, Gentry proposed an efficient identity based encryption with tight security reduction in the standard model but based on a stronger assumption[6].

HIBE. In practice one big organization always has hierarchical structures, perhaps with one central authority, several sub-authorities and many individual users, each belonging to a small part of the organization tree. IBE technique can not directly apply to this situation, we need a solution where each authority can delegate keys to its sub-authorities, who in turn can keep delegating keys further down the hierarchy to the users. hierarchical identity based encryption (HIBE) is such a system. In HIBE cryptosystem, messages are encrypted for identityvectors, representing nodes in the identity hierarchy. In Eurocrypt'02, Horwitz and Lynn [9]

\footnotetext{
${ }^{1}$ This is an independent work with [16]. After we submitting our paper to a journal, we find that work which is available online at April 1th.
} 
first introduced the concept of HIBE, Gentry and Silverberg [8] give the first fully functional HIBE scheme in Asiacrypt'02. But their scheme was only proved secure in the random oracle. Boneh and Boyen [2] first achieved the selective-ID secure efficient HIBE scheme in the standard model in Eurocrypt'04. But the ciphertext length is linear in the depth of the hierarchy. In Eurocrypt'05, Boneh et al. [4] proposed an efficient selective-ID secure HIBE scheme in the standard model with constant size ciphertext. In 2007, Au et al. [1] claimed to construct a HIBE scheme which is fully secure, but later they found a flaw in their security proof. In Informatica 32 (2008), Ren and $\mathrm{Gu}$ [11] claimed to construct a fully secure HIBE scheme with short parameters, high efficiency and tight reduction. Later in Indocrypt'08, they [12] proposed another secure hierarchical identity based encryption scheme with full security in the standard model. But in this paper, we show their schemes are insecure. In TCC'09, Gentry and Halevi [7] proposed fully secure HIBE scheme by using "identity based broadcast encryption with key randomization" (KR-IBBE). In Crypto'09, Waters [15] attained the full security under simple assumption by using "dual system encryption". Recently, Lewko and Waters [10] improved Waters's result to achieve fully secure HIBE with short ciphertexts by using "dual system encryption" in the composite order group.

\subsection{Our Contribution}

We cryptanalysis Ren and Gu's two efficient fully secure HIBE schemes in the standard model. Concretely, from any first level private key, the adversary can easily derive a proper "private key" which can decrypt any ciphertexts for the target identity. That is to say, one query on any first level identity is enough to break their schemes.

\subsection{Organization}

We organize this paper as follows. In section 2, we give the definition and security model for HIBE. In section 3, we review of Ren and Gu's first HIBE scheme and give an attack on it. In section 4, we review of Ren and Gu's second HIBE scheme and give an attack on it. In section 5 , we conclude our paper.

\section{Definition and Security Model}

\subsection{Definition}

A HIBE system consists of the following five algorithms:

$\operatorname{Setup}(\lambda, l)$. Takes as input a security parameter $\lambda$ and the hierarchy depth $l$, it outputs system parameters params and a master secret key $m k$. The system parameters implies also a message space $\mathcal{M}$ (params) and an identity space $\mathcal{I D}$ (params), and hierarchical identities are (ordered) tuples in $\widehat{\mathcal{I D}}$ (params).

KeyGen(params, $m k$, ID). Takes as input the system parameters params and master secret key $m k$, and an identity vector ID $=\left[I D_{1}, \cdots, I D_{t}\right] \in \widehat{\mathcal{I D}}($ params $)$, it outputs a private key $K_{\mathrm{ID}}$ for ID.

KeyDerive(params, ID, $K_{\mathrm{ID}}$, ID'). Takes as input the system parameters params, the identity vector ID and corresponding private key $K_{\mathrm{ID}}$, and another vector ID' such that ID is a prefix of ID', it outputs a private key $K_{\mathrm{ID}^{\prime}}$ for ID'. 
Encrypt(params, ID, $m$ ). Takes as input the system parameters params and identity vector ID and a message $m$, it outputs the the ciphertext $C$.

Decrypt(params, $\left.C, I D, K_{\mathrm{ID}}\right)$. Takes as input the system parameters params, ciphertext $C$, identity vector ID and corresponding private key $K_{\mathrm{ID}}$, it outputs the message $m$ (or an error message $\perp$ ).

\subsection{Security Model}

IND-ID-CCA2 security for HIBE is defined by the following game between an adversary $\mathcal{A}$ and a challenger $\mathcal{B}$.

Setup. The challenger $\mathcal{B}$ runs the Setup algorithm and gives $\mathcal{A}$ the resulting system parameters params, keeping the master key to itself.

Phase 1. $\mathcal{A}$ adaptively issues queries $q_{1}, \cdots, q_{m}$ where query $q_{i}$ is one of the following:

Keygeneration query $\left(\mathrm{ID}_{i}\right) \cdot \mathcal{B}$ responds by running algorithm KeyGen to generate the private key corresponding to $\mathrm{ID}_{i}$ and sends $d_{i}$ to $\mathcal{A}$.

Decryption query $\left(\mathrm{ID}_{i}, c_{i}\right) \cdot \mathcal{B}$ responds by running algorithm KeyGen to generate the private key corresponding to $\mathrm{ID}_{i}$. It then runs algorithm Decrypt to decrypt the ciphertext $c_{i}$ using the private key $d_{i}$ and sends the resulting plaintext to $\mathcal{A}$.

Challenge. $\mathcal{A}$ outputs an identity ID* and two equal length plaintexts $m_{0}, m_{1}$ on which it wishes to be challenged. The only restriction is that $\mathcal{A}$ did not previously issue a key generation query for ID or a prefix of ID. $\mathcal{B}$ picks a random bit $w \in\{0,1\}$ and sends $c$ to $\mathcal{A}$, where $c=\operatorname{Encrypt}\left(\right.$ params, ID, $\left.m_{w}\right)$.

Phase 2. $\mathcal{A}$ issues additional queries $q_{m+1}, \cdots, q_{n}$, where $q_{i}$ is one of:

Keygeneration query $\left(\mathrm{ID}_{i}\right)$ where $I D_{i} \neq I D^{*}$ and $I D_{i}$ is not a prefix of ID*.

Decryption query $\left(\mathrm{ID}_{i}, c_{i}\right)$ where $c_{i} \neq c^{*}$ for $\mathrm{ID}^{*}$ or any prefix of $I D^{*}$.

In both cases, $\mathcal{B}$ responds as in Phase 1 . These queries may be adaptive.

Guess. Finally, the adversary outputs a guess $w^{\prime} \in\{0,1\}$ and wins if $w=w^{\prime}$. We call an adversary $\mathcal{A}$ in the above game an IND-ID-CCA2 adversary. The advantage of $\mathcal{A}$ is defined as $\left|\operatorname{Pr}\left[w=w^{\prime}\right]-\frac{1}{2}\right|$.

Definition 1. An HIBE system is $\left(t, \varepsilon, q_{k}, q_{d}\right)$ IND-ID-CCA2 secure if all $t$-time IND-ID-CCA2 adversaries making at most $q_{k}$ key generation queries and at most $q_{d}$ encryption queries have advantage at most $\varepsilon$ in winning the above game.

\section{Attack on Ren and Gu's First HIBE Scheme}

\subsection{Review of Ren and Gu's First HIBE Scheme}

In this subsection, we review of Ren and Gu's first HIBE scheme in Informatica 32 (2008)[11]

$\operatorname{Setup}(\lambda, l)$. Let $p$ be a large prime number, $G_{1}, G_{2}$ are groups of order $p$. $e: G_{1} \times G_{1} \rightarrow G_{2}$ is a bilinear map, $g$ is a generator of $G_{1}, g_{1}=g^{\alpha}$, where $\alpha \in Z_{p}^{*} . l$ is the maximum number of levels in the HIBE, $H$ is a hash function from $G_{1}^{2} \times G_{1}^{2} \rightarrow Z_{p}^{*}$. The PKG randomly choose $r_{0} \in Z_{p}^{*}, h_{i} \in G_{1}, i=1, \cdots, l$.

$$
\text { params }=\left(g, g_{1}, r_{0}, H, h_{i}(i=0,1, \cdots, l)\right), \quad m k=\alpha
$$


KeyGen(params, $m k$, ID). To a user $U$ with identity $\mathrm{ID}_{i}=\left[I D_{1}, \cdots, I D_{i}\right] \in Z_{p}^{i}$, the PKG randomly choose $r_{i} \in Z_{p}^{*}$, and computes

$$
d_{0, i}=\left(h_{0} g^{-r_{0}}\right)^{\frac{1}{\alpha}} \cdot\left(\prod_{k=1}^{i} h_{k}^{I D_{k}}\right)^{r_{i}}, d_{1, i}=g_{1}^{r_{i}}, d_{i+1, i}=h_{i+1}^{r_{i}}, \cdots, d_{l, i}=h_{l}^{r_{i}}
$$

so the private key of $U$ is $d=\left(d_{0, i}, d_{1, i}, d_{i+1, i}, \cdots, d_{l, i}\right)$.

KeyDerive (params, $\left.\mathrm{ID}_{i-1}, K_{\mathrm{ID}_{i-1}}, \mathrm{ID}_{i}\right)$. The private key for $\mathrm{ID}_{i}=\left[I D_{1}, I D_{2}, \cdots, I D_{i}\right]$ can also be generated by its parent $I \mathrm{D}_{i-1}=\left[I D_{1}, I D_{2}, \cdots, I D_{i-1}\right]$ having the secret key $K_{\mathrm{ID}_{i-1}}=$ $\left(d_{0, i-1}, d_{1, i-1}, d_{i, i-1}, \cdots, d_{l, i-1}\right)$. It computes:

$$
d_{0, i}=d_{0, i-1} \cdot d_{i, i-1}^{I D_{i}} \cdot\left(\prod_{k=1}^{i} h_{k}^{I D_{k}}\right)^{t}, d_{1, i}=d_{1, i-1} \cdot g_{1}^{t}, d_{k, i}=d_{k, i-1} \cdot h_{k}^{t}(k=i+1, \cdots, l)
$$

where $r_{i}=r_{i-1}+t$.

Encrypt(params, ID, $m$ ). To encrypt a message $m \in G_{2}$ for the user with identity $\operatorname{ID}_{i}=$ $\left[I D_{1}, \cdots, I D_{i}\right]$, the sender randomly choose $s \in Z_{p}^{*}$ and compute

$$
c_{1}=\left(\prod_{k=1}^{i} h_{k}^{I D_{k}}\right)^{s}, \quad c_{2}=e(g, g)^{s}, \quad c_{3}=g_{1}^{s}, \quad c_{4}=m \cdot e\left(g, h_{0}\right)^{s}, \quad c_{5}=h_{1}^{s} h_{2}^{s \beta}
$$

where $\beta=H\left(c_{1}, c_{2}, c_{3}, c_{4}\right)$. The ciphertext is $c=\left(c_{1}, c_{2}, c_{3}, c_{4}, c_{5}\right)$.

$\operatorname{Decrypt}\left(\right.$ params, $C$, ID, $\left.K_{\mathrm{ID}}\right)$. The receiver computes $\beta=H\left(c_{1}, c_{2}, c_{3}, c_{4}\right)$, and verifies whether $e\left(g_{1}, c_{5}\right)=e\left(c_{3}, h_{1} h_{2}^{\beta}\right)$. Then he decrypts

$$
m=c_{4} \cdot \frac{e\left(d_{1, i}, c_{1}\right) c_{2}^{-r_{0}}}{e\left(c_{3}, d_{0, i}\right)}
$$

The correctness of their scheme can be verified as follows:

$$
e\left(g_{1}, c_{5}\right)=e\left(g_{1}, h_{1}^{s} h_{2}^{s \beta}\right)=e\left(c_{3}, h_{1} h_{2}^{\beta}\right)
$$

and

$$
\begin{aligned}
& c_{4} \cdot \frac{e\left(d_{1, i}, c_{1}\right) c_{2}^{-r_{0}}}{e\left(c_{3}, d_{0, i}\right)} \\
= & m \cdot e\left(g, h_{0}\right)^{s} \cdot \frac{e\left(g_{1}^{r_{i}}, \prod_{k=1}^{i} h_{k}^{I D_{k}}\right)^{s} e(g, g)^{-s r_{0}}}{e\left(g_{1}^{s},\left(h_{0} g^{-r_{0}}\right)^{\frac{1}{\alpha}} \cdot\left(\prod_{k=1}^{i} h_{k}^{I D_{k}}\right)^{r_{i}}\right)} \\
= & m \cdot e\left(g, h_{0}\right)^{s} \cdot \frac{1}{e\left(g^{s}, h_{0}\right)} \\
= & m
\end{aligned}
$$

\subsection{Our Attack}

1. In the Setup phase, the challenger $\mathcal{B}$ runs the Setup algorithm and gives $\mathcal{A}$ the resulting system parameters params, keeping the master key to itself.

2. In Phase $1, \mathcal{A}$ does not issue any query. 
3. In Challenge phase, $\mathcal{A}$ outputs an identity ID* $=\left[I D_{1}^{*}, I D_{2}^{*}, \cdots, I D_{i}^{*}\right]$ and two equal length plaintexts $m_{0}, m_{1}$ on which it wishes to be challenged. $\mathcal{B}$ picks a random bit $w \in\{0,1\}$ and computes $C^{*}=\operatorname{Encrypt}\left(\right.$ params, ID*,$\left.m_{w}\right)$, sends $C^{*}$ to $\mathcal{A}$. Here

$$
C^{*}=\left(c_{1}=\left(\prod_{k=1}^{i} h_{k}^{I D_{k}^{*}}\right)^{s}, \quad c_{2}=e(g, g)^{s}, \quad c_{3}=g_{1}^{s}, \quad c_{4}=m_{w} \cdot e\left(g, h_{0}\right)^{s}, \quad c_{5}=h_{1}^{s} h_{2}^{s \beta}\right)
$$

where $\beta=H\left(c_{1}, c_{2}, c_{3}, c_{4}\right)$

4. In Phase $2, \mathcal{A}$ does as follows:

(a) First he queries the keygeneration oracle on a first level identity $\mathrm{ID}_{1}=\left[I D_{1}\right], I D_{1} \neq I D_{1}^{*}$ to the challenger $\mathcal{B}$, and $\mathcal{B}$ returns

$$
K_{\mathrm{ID}_{1}}=\left(d_{0,1}=\left(h_{0} g^{-r_{0}}\right)^{\frac{1}{\alpha}} \cdot\left(h_{1}^{I D_{1}}\right)^{r_{i}}, \quad d_{1,1}=g_{1}^{r_{i}}, \quad d_{2,1}=h_{2}^{r_{i}}, \quad \cdots, \quad d_{l, 1}=h_{l}^{r_{i}}\right)
$$

to $\mathcal{A}$.

(b) Then he computes

$$
\begin{aligned}
K_{\mathrm{ID}_{1}}^{\prime}=\left(d_{0,1}^{\prime}=d_{0,1}^{\frac{I D_{1}^{*}}{I D_{1}}}=\left(\left(h_{0} g^{-r_{0}}\right)^{\frac{1}{\alpha}} \cdot\left(h_{1}^{I D_{1}}\right)\right)^{r_{i} \cdot \frac{I D_{1}^{*}}{I D_{1}}}=\left(h_{0} g^{-r_{0}}\right)^{\frac{I D_{1}^{*}}{\alpha I D_{1}}} \cdot\left(h_{1}^{I D_{1}^{*}}\right)^{r_{i}},\right. \\
\left.d_{1,1}^{\prime}=g_{1}^{r_{i}}, d_{2,1}^{\prime}=h_{2}^{r_{i}}, \cdots, d_{l, 1}^{\prime}=h_{l}^{r_{i}}\right)
\end{aligned}
$$

By using the KeyDerive algorithm, he derives a proper "private key"

$$
K_{\mathrm{ID}^{*}}^{\prime}=\left(d_{0, i}^{\prime}=\left(h_{0} g^{-r_{0}}\right)^{\frac{I D_{1}^{*}}{\alpha I D_{1}}}\left(\prod_{k=1}^{i} h_{k}^{I D_{k}^{*}}\right)^{r_{i}^{\prime}}, d_{1, i}^{\prime}=g_{1}^{r_{i}^{\prime}}, d_{i+1, i}^{\prime}=h_{i+1}^{r_{i}^{\prime}}, \cdots, d_{l, i}^{\prime}=h_{l}^{r_{i}^{\prime}}\right)
$$

where $r_{i}^{\prime}$ computed following the KeyDerive algorithm, which is a random element in $Z_{p}^{*}$.

(c) Now he can decrypt the challenge ciphertext $C^{*}$ by using $K_{\mathrm{ID}^{*}}^{\prime}$ as follows

$$
m=c_{4} \cdot\left(\frac{e\left(d_{1, i}^{\prime}, c_{1}\right) c_{2}^{\frac{-r_{0} I D_{1}^{*}}{I D_{1}}}}{e\left(c_{3}, d_{0, i}^{\prime}\right)}\right)^{\frac{I D_{1}}{I D_{1}^{*}}}
$$

We can verify its correctness as follows

$$
\begin{aligned}
& c_{4} \cdot\left(\frac{e\left(d_{1, i}^{\prime}, c_{1}\right) c_{2}^{\frac{-r_{0} I D^{*}}{I D_{1}}}}{e\left(c_{3}, d_{0, i}^{\prime}\right)}\right)^{\frac{I D_{1}}{I D^{*}}} \\
= & m_{w} \cdot e\left(g, h_{0}\right)^{s} \cdot\left(\frac{e\left(g_{1}^{r_{i}^{\prime}}, \prod_{k=1}^{i} h_{k}^{I D_{k}^{*}}\right)^{s} e(g, g)^{\frac{-s r_{0} I D_{1}^{*}}{I D_{1}}}}{e\left(g_{1}^{s},\left(h_{0} g^{-r_{0}}\right)^{\frac{I D_{1}^{*}}{\alpha I D_{1}}} \cdot\left(\prod_{k=1}^{i} h_{k}^{I D_{k}^{*}}\right)^{r_{i}^{\prime}}\right)}\right)^{\frac{I D_{1}}{I D_{1}^{*}}} \\
= & m_{w} \cdot e\left(g, h_{0}\right)^{s} \cdot \frac{1}{e\left(g^{s}, h_{0}\right)} \\
= & m_{w}
\end{aligned}
$$

Obviously, $\mathcal{A}$ wins the IND-ID-CCA2 game with probability 1.

Remark 1. This attack shows that, from any first level private key, it is easy for the adversary to derive a proper "private key" which can decrypt any ciphertexts for the target identity. 


\section{Attack on Ren and Gu's Second HIBE Scheme}

\subsection{Review of Ren and Gu's Second HIBE Scheme}

In this subsection, we review of Ren and Gu's second HIBE scheme in Indocrypt 2008 [12].

$\operatorname{Setup}(\lambda, l)$. Let $p$ be a large prime number, $G_{1}, G_{2}$ are groups of order $p$. e $: G_{1} \times G_{1} \rightarrow G_{2}$ is a bilinear map, $g$ is a generator of $G_{1}, g_{1}=g^{\alpha}$, where $\alpha \in Z_{p}^{*} . l$ is the maximum number of levels in the HIBE, $h$ and $H$ are hash functions $G_{1}^{2} \times G_{2}^{3} \rightarrow Z_{p}^{*}, G_{1}^{2} \times G_{2}^{5} \rightarrow Z_{p}^{*}$. The PKG randomly choose $g_{2}, g_{3}, h_{i} \in G_{1}, i=0,1 \cdots, l$ and $f(x)=a x+b$, where $a, b \in Z_{p}^{*}$. If $g_{2}=g_{3}^{-a}$ or $h_{0}=g_{3}^{-b}$, choose another $f(x)$ again.

$$
\text { params }=\left(g, g_{1}, g_{2}, g_{3}, f(x), h, H, h_{i}(i=0,1, \cdots, l)\right), \quad m k=\alpha
$$

KeyGen(params, $m k$, ID). To a user $U$ with identity $\mathrm{ID}_{i}=\left[I D_{1}, \cdots, I D_{i}\right] \in Z_{p}^{i}$, the PKG randomly choose $r_{-1, i}, r_{0, i} \in Z_{p}^{*}$, and computes

$$
\begin{aligned}
d_{0, i} & =\left(h_{0} g_{2}^{r_{-1, i}} g_{3}^{f\left(r_{-1, i}\right)}\right)^{\alpha} \cdot\left(\prod_{k=1}^{i} h_{l} h_{k}^{I D_{k}}\right)^{r_{0, i}}, d_{-1, i}=r_{-1, i}, d_{1, i}=g^{r_{0, i}}, \\
d_{i+1, i} & =h_{i+1}^{r_{0, i}}, \cdots, d_{l, i}=h_{l}^{r_{0, i}}
\end{aligned}
$$

so the private key of $U$ is $d=\left(d_{0, i}, d_{-1, i}, d_{1, i}, d_{i+1, i}, \cdots, d_{l, i}\right)$. If $h_{0} g_{2}^{r_{-1, i}} g_{3}^{f\left(r_{-1, i}\right)}=1$, randomly choose $r_{-1, i}$ again.

KeyDerive (params, ID $\left.{ }_{i-1}, K_{\mathrm{ID}_{i-1}}, \mathrm{ID}_{i}\right)$. The private key for $\mathrm{ID}_{i}=\left[I D_{1}, I D_{2}, \cdots, I D_{i}\right]$ can also be generated by its parent $\mathrm{ID}_{i-1}=\left[I D_{1}, I D_{2}, \cdots, I D_{i-1}\right]$ having the secret key $K_{\mathrm{ID}_{i-1}}=$ $\left(d_{0, i-1}, d_{-1, i-1}, d_{1, i-1}, d_{i, i-1}, \cdots, d_{l, i-1}\right)$. It computes:

$$
\begin{aligned}
& d_{0, i}=d_{0, i-1} \cdot d_{l, i-1} \cdot d_{i, i-1}^{I D_{i}} \cdot\left(\prod_{k=1}^{i} h_{l} h_{k}^{I D_{k}}\right)^{t}, d_{-1, i}=d_{-1, i-1}, d_{1, i}=d_{1, i-1} \cdot g^{t}, \\
& d_{k, i}=d_{k, i-1} \cdot h_{k}^{t}(k=i+1, \cdots, l)
\end{aligned}
$$

where $r_{0, i}=r_{0, i-1}+t$.

Encrypt(params, ID, $m$ ). To encrypt a message $m \in G_{2}$ for the user with identity $\operatorname{ID}_{i}=$ $\left[I D_{1}, \cdots, I D_{i}\right]$, randomly choose $s \in Z_{p}^{*}$ and compute

$$
\begin{aligned}
& c_{1}=\left(\prod_{k=1}^{i} h_{l} h_{k}^{I D_{k}}\right)^{s}, \quad c_{2}=g^{s}, \quad c_{3}=e\left(g_{1}, g_{2}\right)^{s}, \quad c_{4}=e\left(g_{1}, g_{3}\right)^{s}, \\
& c_{5}=m \cdot e\left(g_{1}, h_{0}\right)^{s+\gamma}, \quad \beta=H\left(c_{1}, c_{2}, c_{3}, c_{4}, c_{5}, m, m \cdot e\left(g_{1}, h_{0}\right)^{s}\right)
\end{aligned}
$$

where $\gamma=h\left(c_{1}, c_{2}, c_{3}, c_{4}, e\left(g_{1}, h_{0}\right)^{s}\right)$. The ciphertext of message $m$ is $c=\left(c_{1}, c_{2}, c_{3}, c_{4}, c_{5}, \beta\right)$. Decrypt(params, $C$, ID, $\left.K_{\mathrm{ID}}\right)$. The recipient computes

$$
\frac{e\left(c_{2}, d_{0, i}\right)}{c_{4}^{f\left(d_{-1, i}\right)} c_{3}^{d_{-1, i}} e\left(c_{1}, d_{1, i}\right)}=e\left(g_{1}, h_{0}\right)^{s}
$$

and

$$
\gamma=h\left(c_{1}, c_{2}, c_{3}, c_{4}, e\left(g_{1}, h_{0}\right)^{s}\right), \quad \frac{c_{5}}{e\left(g_{1}, h_{0}\right)^{\gamma}}=R, \quad \frac{R}{e\left(g_{1}, h_{0}\right)^{s}}=m
$$


Then he computes

$$
\beta^{\prime}=H\left(c_{1}, c_{2}, c_{3}, c_{4}, c_{5}, m, R\right)
$$

and verifies whether $\beta^{\prime}=\beta$, if the equation holds, the ciphertext is valid. Otherwise, the recipient returns an error message.

The correctness of their scheme can be verified as follows:

$$
\begin{aligned}
& \frac{e\left(c_{2}, d_{0, i}\right)}{c_{4}^{f\left(d_{-1, i}\right)} c_{3}^{d_{-1, i}} e\left(c_{1}, d_{1, i}\right)} \\
& =\frac{e\left(g^{s},\left(h_{0} g_{2}^{r_{-1, i}} g_{3}^{f\left(r_{-1, i}\right)}\right)^{\alpha}\left(\prod_{k=1}^{i} h_{l} h_{k}^{I D_{k}}\right)^{r_{0, i}}\right)}{e\left(g_{1}, g_{3}\right)^{s f\left(r_{-1, i}\right)} e\left(g_{1}, g_{2}\right)^{s r_{-1, i}} e\left(\left(\prod_{k=1}^{i} h_{l} h_{k}^{I D_{k}}\right)^{s}, g^{r_{0, i}}\right)} \\
& =e\left(g_{1}, h_{0}\right)^{s}
\end{aligned}
$$

\subsection{Our Attack}

1. In the Setup phase, the challenger $\mathcal{B}$ runs the Setup algorithm and gives $\mathcal{A}$ the resulting system parameters params, keeping the master key to itself.

2. In Phase $1, \mathcal{A}$ does not issue any query.

3. In Challenge phase, $\mathcal{A}$ outputs an identity ID* $=\left[I D_{1}^{*}, I D_{2}^{*}, \cdots, I D_{i}^{*}\right]$ and two equal length plaintexts $m_{0}, m_{1}$ on which it wishes to be challenged. $\mathcal{B}$ picks a random bit $w \in\{0,1\}$ and computes $C^{*}=\operatorname{Encrypt}\left(\right.$ params, ID* $\left.m_{w}\right)$, sends $C^{*}$ to $\mathcal{A}$. Here

$$
\begin{aligned}
C^{*} & =\left(c_{1}=\left(\prod_{k=1}^{i} h_{l} h_{k}^{I D_{k}^{*}}\right)^{s}, \quad c_{2}=g^{s}, \quad c_{3}=e\left(g_{1}, g_{2}\right)^{s}, \quad c_{4}=e\left(g_{1}, g_{3}\right)^{s},\right. \\
c_{5} & \left.=m \cdot e\left(g_{1}, h_{0}\right)^{s+\gamma}, \quad \beta=H\left(c_{1}, c_{2}, c_{3}, c_{4}, c_{5}, m, m \cdot e\left(g_{1}, h_{0}\right)^{s}\right)\right)
\end{aligned}
$$

where $\gamma=h\left(c_{1}, c_{2}, c_{3}, c_{4}, e\left(g_{1}, h_{0}\right)^{s}\right)$.

4. In Phase $2, \mathcal{A}$ does as follows:

(a) First he queries the keygeneration oracle on a first level identity $\mathrm{ID}_{1}=\left[I D_{1}\right], I D_{1} \neq I D_{1}^{*}$ to the challenger $\mathcal{B}$, and $\mathcal{B}$ returns

$$
\begin{aligned}
K_{\mathrm{ID}_{1}}=\left(d_{0,1}\right. & =\left(h_{0} g_{2}^{r_{-1,1}} g_{3}^{f\left(r_{-1,1}\right)}\right)^{\alpha} \cdot\left(h_{l} h_{1}^{I D_{1}}\right)^{r_{0,1}}, d_{-1,1}=r_{-1,1}, d_{1,1}=g^{r_{0,1}}, \\
d_{2,1} & \left.=h_{2}^{r_{0,1}}, \cdots, d_{l, 1}=h_{l}^{r_{0,1}}\right)
\end{aligned}
$$

to $\mathcal{A}$.

(b) Then he computes

$$
\begin{aligned}
T & =\frac{d_{0,1}}{d_{l, 1}}=\left(h_{0} g_{2}^{r_{-1,1}} g_{3}^{f\left(r_{-1,1}\right)}\right)^{\alpha} \cdot\left(h_{1}^{I D_{1}}\right)^{r_{0,1}} \\
K_{\mathrm{ID}_{1}}^{\prime}=\left(d_{0,1}^{\prime}\right. & =T^{\frac{I D_{1}^{*}}{I D_{1}}} \cdot d_{l, 1}=\left(\left(h_{0} g_{2}^{r_{-1,1}} g_{3}^{f\left(r_{-1,1}\right)}\right)^{\alpha} \cdot\left(h_{1}^{I D_{1}}\right)^{r_{0,1}}\right)^{\frac{I D_{1}^{*}}{I D_{1}}} \cdot h_{l}^{r_{0,1}} \\
& =\left(h_{0} g_{2}^{r_{-1,1}} g_{3}^{f\left(r_{-1,1}\right)}\right)^{\frac{\alpha I D_{1}^{*}}{I D_{1}}} \cdot\left(h_{l} h_{1}^{I D_{1}^{*}}\right)^{r_{0,1}}, \\
d_{-1,1}^{\prime} & \left.=r_{-1,1}, d_{1,1}^{\prime}=g^{r_{0,1}}, d_{2,1}^{\prime}=h_{2}^{r_{0,1}}, \cdots, d_{l, 1}^{\prime}=h_{l}^{r_{0,1}}\right)
\end{aligned}
$$


By using the KeyDerive algorithm, he derives a proper "private key"

$$
\begin{gathered}
K_{\mathrm{ID*}}^{\prime}=\left(d_{0, i}^{\prime}=\left(h_{0} g_{2}^{r-1, i} g_{3}^{f\left(r_{-1, i}\right)}\right)^{\frac{\alpha I D_{1}^{*}}{I D_{1}}} \cdot\left(\prod_{k=1}^{i} h_{l} h_{k}^{I D_{k}^{*}}\right)^{r_{0, i}^{\prime}}, d_{-1, i}^{\prime}=r_{-1,1}, d_{1, i}^{\prime}=g^{r_{0, i}^{\prime}},\right. \\
\left.d_{i+1, i}^{\prime}=h_{i+1}^{r_{0, i}^{\prime}}, \cdots, d_{l, i}^{\prime}=h_{l}^{r_{0, i}^{\prime}}\right)
\end{gathered}
$$

where $r_{0, i}^{\prime}$ computed following the KeyDerive algorithm, which is a random element in $Z_{p}^{*}$

(c) Now he can decrypt the challenge ciphertext $C^{*}$ by using $K_{\mathrm{ID}^{*}}^{\prime}$ as follows:

- He first computes

$$
\left(\frac{e\left(c_{2}, d_{0, i}^{\prime}\right)}{\left(c_{4}^{\left.f\left(d_{-1, i}^{\prime}\right) d_{3}^{d_{-1, i}^{\prime}}\right)^{\frac{I D_{1}^{*}}{I D_{1}}} e\left(c_{1}, d_{1, i}^{\prime}\right)}\right.}\right)^{\frac{I D_{1}}{I D_{1}^{*}}}=e\left(g_{1}, h_{0}\right)^{s}
$$

We can verify its correctness as follows

$$
\begin{aligned}
& \left(\frac{e\left(c_{2}, d_{0, i}^{\prime}\right)}{\left(c_{4}^{f\left(d_{-1, i}^{\prime}\right)} c_{3}^{d_{-1, i}^{\prime}}\right)^{\frac{I D_{1}^{*}}{I D_{1}}} e\left(c_{1}, d_{1, i}^{\prime}\right)}\right)^{\frac{I D_{1}}{I D_{1}^{*}}} \\
& =\left(\frac{e\left(g^{s},\left(h_{0} g_{2}^{r-1,1} g_{3}^{f\left(r_{-1,1}\right)}\right)^{\frac{\alpha I D_{1}^{*}}{I D_{1}}}\left(\prod_{k=1}^{i} h_{l} h_{k}^{I D_{k}^{*}}\right)^{r_{0, i}^{\prime}}\right)}{\left(e\left(g_{1}, g_{3}\right)^{s f\left(r_{-1,1}\right)} e\left(g_{1}, g_{2}\right)^{s r_{-1,1}}\right)^{\frac{I D_{1}^{*}}{I D_{1}}} e\left(\left(\prod_{k=1}^{i} h_{l} h_{k}^{I D_{k}^{*}}\right)^{s}, g^{r_{0, i}^{\prime}}\right)}\right)^{\frac{I D_{1}}{I D_{1}^{*}}} \\
& =\left(e\left(g_{1}, h_{0}\right)^{\frac{s I D_{1}^{*}}{I D}}\right)^{\frac{I D_{1}}{I D_{1}^{*}}} \\
& =e\left(g_{1}, h_{0}\right)^{s}
\end{aligned}
$$

- Then he computes

$$
\gamma=h\left(c_{1}, c_{2}, c_{3}, c_{4}, e\left(g_{1}, h_{0}\right)^{s}\right), \quad \frac{c_{5}}{e\left(g_{1}, h_{0}\right)^{\gamma}}=R, \quad \frac{R}{e\left(g_{1}, h_{0}\right)^{s}}=m
$$

Obviously, $\mathcal{A}$ wins the IND-ID-CCA2 game with probability 1.

Remark 2. This attack shows that, from any first level private key, it is easy for the adversary to derive a proper "private key" which can decrypt any ciphertexts for the target identity.

\section{Conclusion}

In this paper, we cryptanalysis two efficient HIBE schemes which claimed to be fully secure in the standard model. Our attacks show that, one key generation query on any first level identity excluding the target's first level identity, is enough to break their schemes.

\section{Acknowledgment}

The authors would like to express their gratitude thanks for Dr. Jun Shao for many helpful comments. 


\section{References}

1. Au, M. H., Liu, J. K., Yuen, T. H., Wong, D.S.: Practical hierarchical identity Based encryption and signature schemes without random oracles. http://eprint.iacr.org/2006/368, 2006.

2. Boneh, D. and Boyen, X.: Efficient selective-id secure identity based encryption without random oracles. EUROCRYPT (C. Cachin, J. Camenisch, Ed.), LNCS 3027, Springer-Verlag, Berlin, 2004.

3. Boneh, D., Boyen, X.: Secure identity based encryption without random oracles. CRYPTO (M. Franklin, Ed.), LNCS 3152, Springer-Verlag, Berlin, 2004.

4. Boneh, D., Boyen, X., Goh, E.: Hierarchical identity based encryption with constant size ciphertext. EUROCRYPT (R. Cramer, Ed.), LNCS 3494, Springer-Verlag, Berlin, 2005.

5. Boneh, D., Franklin., M.: Identity based encryption from the Weil pairing. CRYPTO (J. Kilian, Ed.), LNCS 2139, Springer-Verlag, Berlin, 2001.

6. Gentry, C.: Practical identity-based encryption without random oracles. EUROCRYPT (S. Vaudenay, Ed.), LNCS 4004, Springer-Verlag, Berlin, 2006.

7. Gentry, C., Halevi, S.: Hierarchical identity based encryption with polynomially many levels. TCC (R. Reingold, Ed.), LNCS 5444, Springer-Verlag, Berlin, 2009.

8. Gentry, C., Silverberg, A.: Hierarchical id-based cryptography. ASIACRYPT (Y. Zheng, Ed.), LNCS 2501, Springer-Verlag, Berlin, 2002.

9. Horwitz, J., Lynn, B.: Toward hierarchical identity-based encryption. EUROCRYPT (L.R. Knudsen, Ed.), LNCS 2332, Springer-Verlag, Berlin, 2002.

10. Lewko, A., Waters, B.: Fully secure hibe with short ciphertexts. http://eprint.iacr.org/2009/482, 2009.

11. Ren, Y., Gu, D.: Efficient hierarchical identity based encryption scheme in the standard model. Informatica, 32, 2008, 207-211.

12. Ren, Y., Gu, D.: Secure hierarchical identity based encryption scheme in the standard model. INDOCRYPT (D. R. Chowdhury, V. Rijmen, and A. Das, Ed.), LNCS 5365, Springer-Verlag, Berlin, 2008.

13. Shamir, A.: Identity-based cryptosystems and signature Schemes. CRYPTO (G. R. Blakley and D. Chaum, Ed.), LNCS 196, Springer-Verlag, Berlin, 1984.

14. Waters, B.: Efficient identity-based encryption without random oracles. EUROCRYPT (R. Cramer, Ed.), LNCS 3494, Springer-Verlag, Berlin, 2005.

15. Waters, B.: Dual system encryption: realizing fully secure ibe and hibe under simple assumptions. CRYPTO (S. Halevi, Ed.), LNCS 5677, Springer-Verlag, Berlin, 2009.

16. Weng, J., Chen, M., Chen, K., Deng, R.: Cryptanalysis of a hierarchical identity-based encryption scheme. IEICE Transactions on Fundamentals of Electronics, Communications and Computer Sciences, Volume E93A No.4, 2010, 854-856. 